\title{
Discourse structure and attitudinal valence of opinion words in sentiment extraction
}

\author{
Radoslava Trnavac and Maite Taboada*
}

LSA Annual Meeting, Minneapolis, January 2-5, 2014

1. Introduction. Taboada et al. (2008) propose a word-based method for extracting sentiment from text that relies on the most relevant parts of a text. The method predicts that opinion words found in the nuclei (more important parts) of a document are more significant for the overall sentiment, whereas opinion words found in the satellites (less important parts) only potentially interfere with the overall sentiment. However, as pointed out by Taboada et al. (2008) and Narayanan et al. (2009), for certain discourse relations (for instance, Condition relations), the calculation of sentiment should involve both parts of the relation. Based on our analysis of the affective content expressed by automatically extracted discourse relations from the Simon Fraser University Corpus (Taboada 2008) and the Penn Discourse Treebank (Prasad et al. 2008), we propose to classify all the discourse relations into four categories: (1) relations that reverse polarity, (2) intensify polarity, (3) downtone polarity, or (4) produce no change in polarity. We compare the performance of a sentiment analysis system (SO-CAL, Taboada et al. 2011) when opinion words are detected only in the nuclei with its performance when both parts of the relation are analyzed in combination with the opinion words. The results of the experiment show that extraction of both the nucleus and the satellite parts of texts does not improve the performance of a sentiment extraction system.

\section{Background}

2. 1 PREVIOUS RESEARCH. The area of research that analyzes a relation between discourse structure and attitudinal value of opinion words is relatively new. Taboada et al. (2011) proposed a word-based method for extracting sentiment from texts that should rely on the most relevant parts of texts (nuclei). Heerschop et al. (2011) suggest that there is a possible hierarchy in relations - the satellites of some relations may contribute more to the overall sentiment than others. Asher et al. $(2008,2009)$ found that Result relations strengthen the polarity of the opinion in the second argument, Continue relations strengthen the polarity of the common opinion, while Contrast relations may strengthen or weaken the polarity of opinion expressions. Trnavac and Taboada (2012) analyzed Concessive and Conditional rhetorical relations and concluded that they effect subtle changes in the polarity of the entire sentence (mostly with modified polarity downtoning and intensification).

\footnotetext{
* Radoslava Trnavac, Simon Fraser University (rtrnavac@ @fu.ca) \& Maite Taboada, Simon Fraser University (mtaboada@sfu.ca).
} 
2. 2 RESEARCH QUESTIONS. In this paper we study the following research questions:

1. How do different types of discourse relations in combination with sentiment words influence the evaluative polarity of a relational unit?

2. What is the role of the nucleus-satellite structure in that interaction?

3. Will the performance of a sentiment analysis system (SO-CAL, Taboada et al. 2011) be better when sentiment words are extracted only from the nuclei, or when both parts of the relation are analyzed in combination with the opinion words?

2. 3 PREDICTION. Our prediction is that we can extract opinion words exclusively from the nucleus when the discourse relation reverses polarity or brings no change to polarity.

2.4 THEORETICAL APPROACH. In this paper, we use Rhetorical Structure Theory (Mann \& Thompson 1988; Taboada \& Mann 2006), a theory of discourse coherence. Rhetorical Structure Theory proposes a limited set of relations, such as Cause, Concession, Condition, Elaboration, etc. According to RST, clauses, but also entire sentences, are linked as main and secondary parts. Central spans are marked as nuclei, while less central, or supporting spans, are marked as satellites (Matthiessen \& Thompson 1988).

3. Corpus study. For this study we used the following corpora and software:

- Simon Fraser University Review Corpus (Taboada, 2008). It contains 400 reviews from the website Epinions.com, of movies, books, music, hotels, and consumer products (cars, telephones, cookware and computers) and 281,000 words. We analyzed 11 negative review files from this corpus.

- Penn Discourse Treebank 2.0 (Prasad et al., 2008), a collection of Wall Street Journal articles that has one million words. We analyzed 44 review files.

- A calculator of semantic orientation for words (SO-CAL), with which we extracted words and sentences that express evaluation (Taboada et al., 2011).

3.1 CoRPuS ANNOTATION. We analyzed 848 sentiment words from the SFU Review Corpus (Taboada, 2008) and 850 sentiment words from the Penn Discourse Treebank 2.0 (Prasad et al., 2008), and focused on eight discourse relations.

The following five parameters were included in corpus annotation:

- Type of a relation (or sense) in which the word occurs.

- Position of the word within nucleus-satellite (Arg1/Arg2) structure.

- Positive or negative polarity of the word.

- Word class.

- Polarity formed between the two parts of a relation (two arguments of a sense) - reversal, intensification, downtoning and no change of polarity. 


\subsection{ANALYSIS OF CORPORA AND RESULTS.}

\begin{tabular}{|l|c|l|c|}
\hline Relations (SFU) & Number of words & \multicolumn{1}{c|}{ Sense (PDTB) } & Number of words \\
\hline Background & 22 & Pragmatic cause & 4 \\
\hline Cause & 28 & Cause-result & 172 \\
\hline Circumstance & 34 & Temporal & 73 \\
\hline Concession & 63 & Concession & 48 \\
\hline Contrast & 33 & Contrast & 95 \\
\hline Elaboration & 51 & EntRel & 29 \\
\cline { 2 - 4 } & & Expansion: Instantiation & 101 \\
\cline { 2 - 4 } & & Expansion: Restatement & 229 \\
\hline Joint & 74 & Expansion: Conjunction & - \\
\hline Purpose & 9 & - & \\
\hline
\end{tabular}

Table 1. Mapping of relations (SFU Corpus and PDTB)

\begin{tabular}{|l|c|c|c|c|}
\hline \multicolumn{1}{|c|}{ Relations (N/S) } & Reversal & Downtoning & Intensification & No change \\
\hline Background & $5 / 1$ & $1 / 0$ & $1 / 1$ & $7 / 6$ \\
\hline Cause & $1 / 0$ & $2 / 0$ & $1 / 3$ & $14 / 7$ \\
\hline Circumstance & $4 / 1$ & $1 / 0$ & $13 / 15$ & $0 / 0$ \\
\hline Concession & $27 / 23$ & $3 / 1$ & $1 / 0$ & $4 / 4$ \\
\hline Contrast & $19 / 0$ & $0 / 0$ & $4 / 0$ & $10 / 0$ \\
\hline Elaboration & $3 / 1$ & $2 / 1$ & $5 / 3$ & $19 / 17$ \\
\hline Joint & $9 / 0$ & $0 / 0$ & $17 / 0$ & $48 / 0$ \\
\hline Purpose & - & - & - & $4 / 5$ \\
\hline
\end{tabular}

Table 2. Rhetorical relations with nucleus-satellite structure and polarity (SFU Corpus)

\begin{tabular}{|l|c|c|c|c|}
\hline \multicolumn{1}{c|}{$\begin{array}{c}\text { Relations } \\
\text { Arg1/Arg2) }\end{array}$} & Reversal & Downtoning & Intensification & No change \\
\hline Cause-Result & $9 / 4$ & - & - & $84 / 75$ \\
\hline Concession & $16 / 12$ & - & - & $4 / 16$ \\
\hline Contrast & $43 / 38$ & - & - & $10 / 4$ \\
\hline EntRel & $8 / 8$ & - & - & $34 / 30$ \\
\hline $\begin{array}{l}\text { Expansion: } \\
\text { Conjunction }\end{array}$ & $16 / 10$ & - & $27 / 20$ & $77 / 79$ \\
\hline $\begin{array}{l}\text { Expansion: } \\
\text { Instantiation }\end{array}$ & $0 / 3$ & - & $0 / 3$ & $14 / 9$ \\
\hline $\begin{array}{l}\text { Expansion: } \\
\text { Restatement }\end{array}$ & $2 / 4$ & - & $1 / 1$ & $52 / 41$ \\
\hline Pragmatic cause & - & - & - & $3 / 1$ \\
\hline Temporal & $7 / 3$ & - & $2 / 1$ & $29 / 31$ \\
\hline
\end{tabular}

Table 3. Senses with the Arg1-Arg2 structure and polarity of evaluation (PDTB) 


\section{SO-CAL experiment}

4.1 EXPERIMENT. We extracted sentiment using the SO-CAL software to determine whether a text is mostly positive or negative, and using two settings: entire text (raw), or only nucleus/Arg1.

4.2 RESULTS. There is no significant difference in the performance of SO-CAL when using only nuclei as compared to using both nuclei and satellites. In fact, in the PDTB, the performance declines when using only Arg1.

\begin{tabular}{|c|c|c|}
\hline \multicolumn{1}{|c|}{ Corpus } & Raw text results & Only nuclei results \\
\hline SFU Review Corpus & $90.9 \%$ & $90.9 \%$ \\
\hline PDTB & $72.7 \%$ & $59.1 \%$ \\
\hline
\end{tabular}

Table 4. SO-CAL results for SFU and PDTB corpora

\section{Discussion and conclusions}

- "No change" is the most frequent type of polarity for all types of relations, with the exception of the Concessive and Contrast relations (reversed polarity) in both corpora.

- In the SFU Review corpus, sentiment words are slightly more frequent in the nucleus of the most common relations such as Concession, Circumstance, Cause and Elaboration, while in Contrast and Joint, the structure consists of the nuclei by default.

- Since "No change" is the most frequent type of polarity, nuclei occur more often with "No change" polarity, except for Concession (reversed polarity).

- In the PDTB corpus, sentiment words are slightly more frequent in the most common relations such as Restatement, Conjunction, Instantiation, EntRel, while in Contrast the structure consists of the nuclei by default.

- Since "No change" of polarity is the most frequent type of polarity in our corpora, the result of the SO-CAL experiment is unsurprising - there is no significant difference in performance of a sentiment analysis system when using only nuclei as opposed to using both nuclei and satellites. The polarity expressed with sentiment words in the nuclei is not affected by the sentiment words in the satellites.

- The aim of future research is to classify existing types of discourse relations in their interaction with subjective information. We plan to extend our corpus analysis to all RST-like relations and show statistical tendencies for attraction between polarity types, types of relations and positive/negative sentiment words. 


\section{References}

Asher, Nicholas, Benamara, Farah, \& Mathieu, Yvette Yannick. (2008). Distilling opinion in discourse: A preliminary study. Proceedings of COLING (pp. 7-10). Manchester, UK.

Asher, Nicholas, Benamara, Farah, \& Mathieu, Yvette Yannick. (2009). Appraisal of opinion expressions in discourse. Linguisticae Investigationes, 32(2), 279-292.

Heerschop, Bas, Goosen, Frank, Hogenboom, Alexander, Frasincar, Flavius, Kaymak, Uzay, \& de Jong, Franciska. (2011). Polarity analysis of text using discourse structure.

Proceedings of the 20th ACM International Conference on Information and Knowledge Management (CIKM2011), (pp. 1061-1070). Glasgow, UK.

Mann, William C., \& Thompson, Sandra A. (1988). Rhetorical Structure Theory: Toward a functional theory of text organization. Text, 8(3), 243-281.

Matthiessen, Christian M.I.M., \& Thompson, Sandra A. (1988). The structure of discourse and "subordination". In J. Haiman \& S. A. Thompson (Eds.), Clause Combining in Discourse and Grammar (pp. 275- 329). Amsterdam and Philadelphia: John Benjamins.

Narayanan, Ramanathan, Liu, Bing, and Alok Choudhary (2009). Sentiment Analysis of Conditional Sentences. Proceedings of the 2009 Conference on Empirical Methods in Natural Language Processing, pages 180189.

Prasad, Rashmi, Lee, Alan, Dinesh, Nikhil, Miltsakaki, Eleni, Campion, Geraud, Joshi, Aravind K., \& Webber, Bonnie. (2008). Penn Discourse Treebank Version 2.0, LDC2008T05 [Corpus]. Philadelphia, PA: Linguistic Data Consortium.

Taboada, Maite. (2008). SFU Review Corpus [Corpus]. Vancouver: Simon Fraser University,

http://www.sfu.ca/ mtaboada/research/SFU_Review_Corpus.html.

Taboada, Maite, \& Mann, William C. (2006). Rhetorical Structure Theory: Looking back and moving ahead. Discourse Studies, 8(3), 423-459.

Taboada, Maite, Kimberly Voll and Julian Brooke (2008). Extracting Sentiment as a Function of Discourse Structure and Topicality. School of Computing Science Technical Report 2008-20.

Taboada, Maite, Brooke, Julian, Tofiloski, Milan, Voll, Kimberly, \& Stede, Manfred. (2011). Lexicon-based methods for sentiment analysis. Computational Linguistics, 37(2), 267-307.

Trnavac, Radoslava, \& Taboada, Maite. (2012). The contribution of nonveridical rhetorical relations to evaluation in discourse. Language Sciences, 34(3), 301-318. 